\title{
IL RUOLO DELLE RELAZIONI TRA PARI NELLA COSTRUZIONE DELLA QUALITÀ DI VITA NEI RAGAZZI CON DIAGNOSI DI TUMORE
} \author{
Gruppo di Lavoro Adolescenti AIEOP A
Indirizzo per corr
THE ROLE OF PEER RELATIONSHIPS
IN CONSTRUCTING QUALITY OF LIFE \\ IN ADOLESCENTS WITH CANCER
}

Key words

Adolescent, Cancer, Friends, Peer, Quality of life

\begin{abstract}
Introduction - The optimization of cancer care in adolescent or young adult patients (AYA) requires the contribution of the patients themselves, as they are builders and custodians of the experience that concerns them. A more multidimensional listening to these patients guarantees a better quality of life, in response to their specific needs. The present article describes how a congress between doctors, psychologists, nurses and AYA patients can be the context of exploration and collection of this important contribution. By offering these patients the opportunity to express thoughts, emotions and needs in terms of quality of life verbally and freely, such a congress becomes a privileged place for observation and analysis even of macro-group dynamics that otherwise would not have the possibility of making themselves visible.
\end{abstract}

Methods - Through a qualitative research design, 35 Italian cancer patients (in active treatment or follow-up) aged between 18 and 24 years were involved in a plenary discussion during a congress (Rome, 11 November 2019). The structured interview asked them to express themselves on the issue of quality of life connected to the relationship dynamics with peers. The 55 responses collected were then subjected to thematic analysis.

Results - The young people who attended expressed precise and extensive considerations regarding friendships and the value of interactions with patients of the same age. What emerged enabled to highlight the need of these patients to keep active the social group of reference and in particular the identity of each within it. This is significantly connected to the self-perceived quality of life, both during active treatments and afterwards (a phase described as complex and tiring).
Discussion - The described experience underlines the importance for AYA cancer patients to express their reflections on health and quality of life issues. The relational dimension, especially regarding peers, is significantly connected to this, to one's perception of identity and to the configuration of the "disease" reality, so health personnel are required to protect and attend to this aspect as one of the priorities of the treatment path. The setting offered by the congress also allowed personal expression even in the face of a group recognition that moves the subject from the Ego to the Us.

\section{RIASSUNTO}

Introduzione - Nell'ottica di una ottimizzazione della cura oncologica nei pazienti adolescenti o giovani adulti (AYA) rientra anche la fase dell'ascolto del paziente stesso come depositario di una storia particolare, anche se colpito da una patologia universale. In questo articolo si riporta l'esperienza di un congresso tra medici, psicologi, infermieri e pazienti AYA, divenuto momento di esplorazione e di raccolta delle testimonianze libere dei ragazzi.

Metodi - Mediante un disegno di ricerca qualitativa, nel congresso (Roma, 11 novembre 2019) sono stati coinvolti, in una discussione plenaria, 35 pazienti oncologici italiani (in trattamento attivo o follow-up) di età compresa tra $18 \mathrm{e}$ 24 anni. L'intervista strutturata ha permesso loro di esprimersi circa il tema della qualità di vita connessa alle dinamiche relazionali con i pari. Le 55 risposte raccolte sono state poi sottoposte ad analisi tematica.

Risultati - I ragazzi intervenuti hanno espresso considerazioni puntuali ed estese in merito alle relazioni amicali e al valore delle interazioni con i pazienti coetanei. Quanto emerso ha messo in luce il bisogno dei pazienti di mantenere attivo il gruppo sociale di riferimento e in particolare l'identità di ciascuno all'interno dello stesso. Tale elemento è significativamente connesso alla qualità di vita autopercepita, sia durante i trattamenti attivi, sia in seguito (fase descritta come complessa e faticosa).

Discussione - Questa esperienza ha sottolineato l'importanza, per i pazienti oncologici AYA, di esprimere le proprie riflessioni sugli argomenti riguardanti la salute e la qualità di vita. La dimensione relazionale, soprattutto tra 
pari, è significativamente connessa alla propria percezione identitaria e alla configurazione della realtà "malattia": questo richiede al personale sanitario di tutelare e attendere a tale aspetto come tra $\mathrm{i}$ prioritari del percorso di cura. Il congresso, favorendo lo spostamento del soggetto dall'Io al Noi, è divenuto luogo privilegiato di osservazione e analisi anche di dinamiche macrogruppali alle quali la malattia come esperienza del singolo non consente di avere accesso.

La cosa importante, secondo me, non erano tanto $i$ messaggi, "come stai?", ma piuttosto come ti sentivi con loro... (Giulia, 22 anni)

È stata proprio questa la mia forza, ciò̀ che io personalmente ho avuto la fortuna di avere degli amici sempre presenti. (David, 18 anni)

\section{INTRODUZIONE}

Gli adolescenti e i giovani adulti (AYA) con tumore affrontano sfide mediche e psicosociali peculiari, che li differenziano dai pazienti più anziani e dalla popolazione pediatrica. Ogni anno, in Italia, a circa 800 adolescenti e a 1100 giovani adulti, di età compresa rispettivamente tra 15 e 19 anni e tra 20 e 24 anni, viene posta una diagnosi di tumore ${ }^{1}$.

Nei Paesi occidentali, fin dai primi anni Duemila, per le specificità di questo gruppo di pazienti e per i mancati progressi nelle percentuali di sopravvivenza a confronto di altre fasce di età, sono stati sviluppati programmi loro dedicati, che hanno coinvolto differenti operatori sanitari, vari portatori di interesse e ovviamente i pazienti stessi ${ }^{2-6}$.

\begin{tabular}{|c|c|}
\hline \multicolumn{2}{|r|}{ OBIETTIVI E PROGETTUALITÀ DEL GRUPPO DI LAVORO ADOLESCENTI AIEOP } \\
\hline Obiettivo & Descrizione \\
\hline Culturale & $\begin{array}{l}\text { Promuovere la conoscenza delle problematiche dei tumori in adolescenza tra i professionisti } \\
\text { della sanità e nella popolazione. }\end{array}$ \\
\hline Organizzativo & $\begin{array}{l}\text { Promuovere lo sviluppo nel territorio Italiano di una serie di Centri con spazi dedicati e } \\
\text { personale formato per la cura degli adolescenti con tumore. }\end{array}$ \\
\hline Formativo & $\begin{array}{l}\text { Favorire la formazione specifica del personale medico, infermieristico, psicologico, } \\
\text { educazionale e di supporto. }\end{array}$ \\
\hline Reclutamento & $\begin{array}{l}\text { Evitare la dispersione degli adolescenti oncologici in Centri con scarsa esperienza per queste } \\
\text { patologie e privi di progettualità dedicate. }\end{array}$ \\
\hline Terapeutico & $\begin{array}{l}\text { Migliorare e incrementare la percentuale di arruolamento di questi pazienti, affetti da malattie } \\
\text { rare, in clinical trial multicentrici. } \\
\text { Applicare protocolli di cura che abbiano tra gli obiettivi principali non solo la guarigione, ma } \\
\text { anche la riduzione degli effetti collaterali a lungo termine secondari alle terapie. } \\
\text { Garantire nel maggior numero di pazienti, compatibilmente con le condizioni cliniche, } \\
\text { procedure e metodiche per la preservazione della fertilità. }\end{array}$ \\
\hline Collaborativo & $\begin{array}{l}\text { Migliorare la collaborazione con l'oncologia e le società scientifiche oncologiche dell'adulto } \\
\text { per lo sviluppo di protocolli e progetti condivisi, non limitati all'adolescente propriamente det- } \\
\text { to (15-19 anni), ma anche al giovane adulto (20-25 anni), paziente con bisogni assistenziali e } \\
\text { istotipi tumorali analoghi. }\end{array}$ \\
\hline Sociale & $\begin{array}{l}\text { Valorizzare iniziative (sociali, ludiche, scolastiche) dedicate ai pazienti adolescenti e ai giovani } \\
\text { malati di tumore. } \\
\text { Migliorare il rapporto e il coinvolgimento dei coetanei sani, per favorire il pieno reintegro } \\
\text { sociale, scolastico o lavorativo. } \\
\text { Collaborare con le associazioni genitori e con le associazioni pazienti per la promozione e il } \\
\text { finanziamento della ricerca. }\end{array}$ \\
\hline Ricerca & $\begin{array}{l}\text { Favorire il collegamento con strutture internazionali, per implementare la cooperazione e la } \\
\text { ricerca con altri stati e altri gruppi di lavoro. } \\
\text { Stimolare la pubblicazione scientifica dei risultati, dei progetti e delle collaborazioni realizzate } \\
\text { dal Gruppo di Lavoro Adolescenti AIEOP. }\end{array}$ \\
\hline Follow-up & $\begin{array}{l}\text { Implementare e favorire l'applicazione di percorsi clinico assistenziali, in accordo con la } \\
\text { Medicina di territorio, per il monitoraggio a lungo termine dei possibili effetti collaterali. }\end{array}$ \\
\hline
\end{tabular}

Tabella I 
Il riconoscimento di una diagnosi oncologica pone in essere la presa in carico della persona da parte di una struttura sanitaria appropriata, al fine di eradicare/contenere il più possibile la malattia, moderare le sue sequele, riconfigurare il processo evolutivo dell'interessato e offrire i supporti clinici necessari per costruire/ripristinare la salute globale dello stesso. All'interno di tale prospettiva, che ha come protagonista un adolescente, la caring challenge assume coordinate tipicamente orientate, tanto da richiedere un'attenzione ancor più sostenuta ed esperta circa i processi comunicativi e relazionali medico-paziente ${ }^{6,7}$. Di conseguenza, la costruzione del ruolo attivo dell'individuo nel proprio percorso terapeutico e nella continuità della propria vita extraospedaliera diviene il precursore di una maggiore compliance, di un migliore adattamento e di un più alto livello di benessere psicologico. A partire dalle prime tappe del percorso, la diagnosi di cancro si configura come un evento critico che frantuma la propria biografia, minacciando l'individuo e travolgendo la dimensione fisica, psicologica e relazionale 8,9 .

Il convegno denominato "Adolescenti e tumore", svoltosi a Roma il 9 novembre 2019, ha fatto da contenitore all'indagine - di cui qui se ne descrive una parte - che aveva come obiettivo lo studio degli aspetti relativi alla qualità di vita degli adolescenti con tumore. Tale evento è stato organizzato dal Gruppo di Lavoro Adolescenti della Associazione Italiana di Emato-Oncologia Pediatrica (AIEOP), Gruppo di Lavoro atto a migliorare la qualità della cura e a promuovere i diversi ambiti di ricerca biologica, clinica e psicologica in questo set particolare di pazienti (Tabella I).

Questo contributo si propone di entrare nel merito della sfera relazionale dei pazienti AYA durante la malattia e i trattamenti, al fine di descrivere e comprenderne gli aspetti salienti, i facilitatori, le criticità e le peculiarità. Si intende, in particolare, descrivere il ruolo significativo dei pari (amici noti e nuovi, e altri pazienti coetanei) in riferimento a resoconti, bisogni e richieste, impattanti la qualità di vita nel percorso di cura.

\section{MATERIALI E METODI}

Oltre a medici, infermieri, psicologi e rappresentanti delle associazioni genitori, hanno partecipato al Convegno 35 pazienti adolescenti con tumore, di età compresa tra i 18 e i 24 anni, provenienti da 15 differenti Centri AIEOP. Il $25 \%$ era in trattamento attivo, il $75 \%$ in followup. Durante la tavola rotonda pomeridiana, organizzata insieme ai giovani partecipanti, è stata condotta un'intervista plenaria semi-strutturata che si proponeva di raccogliere le percezioni dei giovani pazienti circa i processi interattivi avvenuti durante i percorsi di diagnosi e di cura.

L'intervista è stata confezionata mediante un approccio narrativo, modello operativo che consente di comprendere gestire le difficoltà caratteristiche della comunicazione in plenaria e di non incardinare le risposte dei ragazzi in schemi precostituiti. Tale scelta ha reso necessario l'inquadramento sperimentale dell'indagine nei ranghi della ricerca qualitativa, che ha così offerto ai partecipanti la maggiore libertà espressiva possibile. Ciascun ragazzo era stato precedentemente informato delle specifiche di indagine, nonché della sua audioregistrazione, e vi ha dunque partecipato previo consenso (Figura 1).

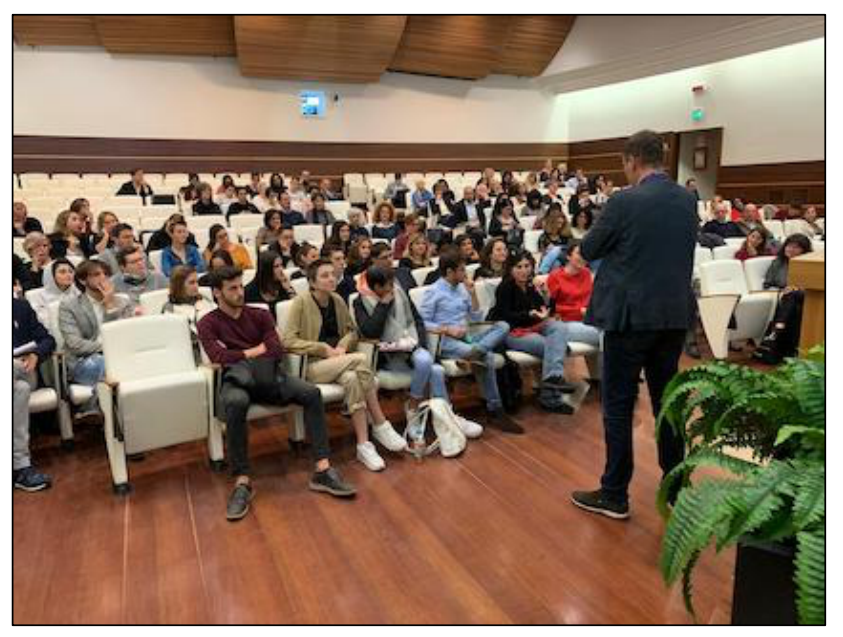

Figura 1. I ragazzi rispondono in plenaria, di fronte a medici, infermieri e psicologi, alle domande sulla comunicazione della diagnosi.

Il questionario, costruito ad hoc, si componeva di 14 domande centrate sulle molteplici sfaccettature delle interazioni interpersonali in oncologia durante le cure, ovvero medico/operatore $\mathrm{n}$ paziente $\mathrm{n}$ caregiver $\mathrm{n}$ amici, evocando tematiche considerate dalla letteratura rilevanti e caratteristiche dell'età anagrafica dei rispondenti. In questo specifico contributo si riportano soltanto le domande relative alla dimensione relazionale e amicale, con i pari, ambito peculiare nel quale i ragazzi riconoscono gli indicatori della qualità di vita autopercepita (Tabella II).

Per ciascun item è stato definito un tempo massimo di interlocuzione di 180 secondi ed è stato lasciato libero diritto di intervento a ciascun partecipante, mediante alzata di mano. Quando nessuno dei ragazzi partecipanti aveva da aggiungere qualcosa si procedeva all'item successivo. Poste una alla volta ed enunciate da un medico (referente di struttura di uno dei Centri coinvolti), le domande sono state proiettate sullo schermo della sala congressuale, e, laddove necessario, commentate da un altro medico specializzato in Psicologia clinica e in Psicoterapia, preposto a contenere eventuali precipitati emotivi e narrativi.

L'intera sessione operativa è stata successivamente trascritta per una lettura e uno studio dettagliato dei testi in riferimento agli obiettivi specifici ${ }^{10}$. I risultati riportati sono rappresentativi di quanto emerso dall'analisi delle 55 risposte prodotte dai ragazzi sulla loro sfera relazionale, rispetto alla quale non si è ancora indagato molto. 


\section{ALCUNE DOMANDE DELL'INTERVISTA STRUTTURATA PER RACCOGLIERE LE ESPERIENZE DEI PAZIENTI, RELATIVAMENTE ALLA DIAGNOSI E ALLE RELAZIONI CON I PARI}

\begin{tabular}{|l|l|}
\hline Domanda & Argomento \\
\hline $\begin{array}{l}\text { 3. Qual è la prima persona, oltre ai tuoi genitori, alla } \\
\text { quale l'hai comunicato? Come l'hai comunicato... tele- } \\
\text { fono, social, dal vivo? }\end{array}$ & $\begin{array}{l}\text { Le azioni comunicative agite di conseguenza nei con- } \\
\text { fronti di terzi non familiari. }\end{array}$ \\
\hline $\begin{array}{l}\text { 4. E gli amici? Come si sono posti in tutto questo? } \\
\begin{array}{l}\text { 8. Qualcuno ha superato i confini che ti eri posto intor- } \\
\text { no? Ti ha dato fastidio o hai gradito questa cosa? }\end{array}\end{array}$ & $\begin{array}{l}\text { La configurazione delle relazioni amicali durante il } \\
\text { percorso. Definizioni e posizionamenti. }\end{array}$ \\
\hline $\begin{array}{l}\text { 11. Avresti voluto parlare con qualcuno che ha vissuto } \\
\text { la tua stessa esperienza? }\end{array}$ & $\begin{array}{l}\text { Il ruolo derapie come eventi che richiedono la } \\
\text { anagrafiche e medicali. }\end{array}$ \\
\hline $\begin{array}{l}\text { 14. Come è cambiato il rapporto con te stesso, nel fisi- } \\
\text { co e nel carattere? Te ne sei accorto da solo o qualcuno interpersonali. } \\
\text { te l'ha fatto notare? }\end{array}$ & $\begin{array}{l}\text { Il rapporto con il sé corporeo e caratteriale in seguito ai } \\
\text { trattamenti. }\end{array}$ \\
\hline
\end{tabular}

\section{Tabella II}

\section{RISULTATI}

Sin dalla prima enunciazione, ogni domanda ha prodotto una spontanea disposizione dei presenti a esprimersi in prima persona. Ogni risposta ha esordito con un resoconto più o meno puntuale della propria storia di malattia, costituita da eventi rilevanti, attori molteplici, emozioni semplici e complesse.

Entrando nel merito dei nuclei tematici connessi alle relazioni con i pari, si riportano a titolo esemplificativo alcuni dei testi ottenuti (Tabella III).

Il tema a cui i ragazzi hanno dedicato più spazio è stato quello dell'amicizia. Circa il 75\% avrebbe desiderato intervenire e tutti coloro a cui è stata data voce (6 rispondenti) hanno suddiviso gli amici in due categorie: chi ha saputo accompagnare e rendersi presente durante l'intero percorso e chi, nel tempo, ha allentato la qualità e le occasioni della relazione.

Cinque ragazzi su sei hanno rilevato un cambiamento nella qualità di vita percependo modificate la frequenza delle interazioni e le caratteristiche delle loro amicizie. In particolare, hanno narrato come durante la malattia i rapporti che meglio si sono adattati a incombenze, bisogni e comprensioni reciproche, sono stati mantenuti.

I pazienti che hanno percepito su di sé l'attribuzione di "malato" o "vittima" hanno descritto due scenari: alcuni hanno riferito di aver ricevuto un maggior accudimento (2 su 6), mentre altri (4 su 6) - e tutti a lungo termine - si sono autodefiniti "impotenti" e "troppo seri".

Subito di seguito ai genitori (in particolare alla madre, vissuta come il più grande "aiuto") e ai fratelli, la prima persona a cui i giovani pazienti si sono trovati nell'urgenza di comunicare la propria diagnosi di malattia appena ricevuta è stato proprio il "migliore amico", raggiunto in fretta e tramite smartphone (3 rispondenti su 3).

Il ruolo degli "altri pazienti", ovvero di quei coetanei che più o meno contemporaneamente affrontavano simili percorsi di malattia, è stato riconosciuto "utile" e addirittura "insostituibile", soprattutto quando potevano anticipare circostanze ed eventi del percorso stesso. Quelle relazioni sono state descritte come capaci di offrire uno spazio importante, libero e autentico, di rielaborazione degli accadimenti, che ha chiarito i significati degli eventi e delle indicazioni medico-infermieristiche. Dal punto di vista psicologico, "il dopo" - ovvero la fase di follow-up - è stato descritto "difficile" quanto o addirittura di più rispetto al periodo delle cure: i ragazzi hanno impiegato pensieri ed energie nell'affrontare un percorso postumo obbligato e inevitabile, proprio perché connotato dalla difficoltà a ri-costruire e/o ri-affrontare la dimensione relazionale e gruppale della propria vita. La fine delle terapie porta con sé molte incertezze, frutto anche dell'allargamento o della recisione delle maglie familiari e sanitarie, supporto forse non più così necessario. Essa è spesso descritta come manchevole di "indicazioni”, di prospettive solide e di chiarezze. Riprendere la quotidianità è "impegnativo".

I ragazzi configurano la qualità e la quantità delle amicizie come indicatore significativo della propria qualità di vita. A trattamenti conclusi, si descrivono "spaesati", "cambiati" e dicono, in particolare, di "percepire" diversamente rispetto a prima il mondo esterno - relazionale e simbolico - oltre a sentire l'indeterminatezza di sé, connessa alla percezione del rischio di recidiva. Sulla base di tali elementi, qualcuno di loro ha definito questa fase temporale con l'espressione "periodo depressivo". 


\section{TRASPOSIZIONE DI STRALCI DI TESTI DEI RISPONDENTI SUDDIVISI PER AREE DI CONTENUTO}

\begin{tabular}{|c|c|}
\hline Item 3 & $\begin{array}{l}\text { "la prima persona a cui l'ho comunicato sono stati i miei migliori amici, e l'ho detto attraverso } \\
\text { WhatsApp ..." (Mari, } 20 \text { anni) }\end{array}$ \\
\hline Item 4-14 & $\begin{array}{l}\text { "Ci sono due tipi di amici. Quelli che all'inizio ti stanno molto vicino, ti scrivono ogni giorno, ti } \\
\text { chiedono come stai, chiedono informazioni ... e poi con l'andare del tempo non vieni a sapere } \\
\text { più niente di loro. E ci sono gli amici, quelli veri, quelle persone che nonostante tutto, nonostante } \\
\text { mi potessi arrabbiare con loro perché avevo bisogno di sfogarmi per qualsiasi motivo, ci sono } \\
\text { sempre, ti sostengono e sono sempre accanto a te." (Alessandro, } 20 \text { anni) } \\
\text { "Al termine delle terapie mi sentivo un'altra... non riuscivo più a interagire con queste persone, } \\
\text { o non più come volevo prima. Tu sei andato avanti, e gli altri sono andati avanti un po' meno } \\
\text { veloci di te, ..." (Anna } 19 \text { anni) }\end{array}$ \\
\hline Item 5 & $\begin{array}{l}\text { "Ci sono stati due momenti di forte paura. All'inizio, quando hai la diagnosi: lì tutto si blocca, } \\
\text { cioè proprio come un secchio di acqua gelata in testa e dici -E adesso, che faccio? E alla fine, } \\
\text { quando devi riprendere la quotidianità, quando ti sei estraniato e vivi in un mondo parallelo" } \\
\text { (Stefano, } 19 \text { anni) }\end{array}$ \\
\hline Item 11 & $\begin{array}{l}\text { “... mi fidavo più dei ragazzi perché sapevano parlare un po' la mia lingua, con me, che avevo la } \\
\text { loro età, rispetto ai dottori.” (Erika, } 18 \text { anni) } \\
\text { “... quei } 5 \text { minuti sono stati molto importanti, perché mi hanno dato gli strumenti per potermi } \\
\text { confrontare con qualcuno che stava passando la mia stessa malattia. E la seconda volta che ci } \\
\text { siamo incontrate praticamente eravamo amiche nonostante ci fossimo effettivamente viste solo } 5 \\
\text { minuti.” (Anna, } 18 \text { anni) }\end{array}$ \\
\hline Item 14 & $\begin{array}{l}\text { “... non avevo nessuna voglia di stare lì dentro, di socializzare con gli altri ragazzi. Io mi sono } \\
\text { chiusa completamente a riccio senza voler avere nulla a che fare con quelle persone. E qui salta } \\
\text { fuori Matteo, l'educatore. Lui è stato veramente importante: è riuscito a prendermi, a tirarmi } \\
\text { fuori da questo riccio." (Cecilia, } 19 \text { anni) }\end{array}$ \\
\hline
\end{tabular}

Tabella III

\section{DISCUSSIONE}

La voce dei ragazzi ha descritto in termini specifici e altamente competenti cosa accade in termini emotivi, cognitivi ed esperienziali nella dimensione relazionale che è loro propria: quella con i pari.

Mostrandosi come altamente significativa, la questione del mantenimento delle relazioni amicali rappresenta un nucleo tematico e clinico da considerare con priorità ${ }^{11-13}$. La disponibilità di uno spettro relazionale con i pari sottende, per il giovane paziente, una valutazione addirittura identitaria, che lo porta a configurarsi nei termini delle attribuzioni poste da altri. Fronteggiare la malattia avendo a fianco "qualcuno che parla la tua stessa lingua", sia dentro che fuori dall'ospedale (amici noti e pazienti coetanei), permette a chi è sottoposto alle cure di mantenere un ruolo sociale connotato positivamente e di comprendere meglio lo stato dell'arte delle indicazioni mediche, dei trattamenti e degli effetti collaterali. Vi è la necessità, per il personale dedicato a questo gruppo di pazienti e per i caregiver, di volgere le attenzioni a questo tema specifico, per loro prioritario. Chiunque si occupi di attività clinica deve avere ben presente due aspetti: quello del punto di vista del medico e quello del punto di vista del paziente ${ }^{14}$.

La qualità di vita del giovane paziente non può eludere le dinamiche amicali, motivo per cui gli va offerto un supporto psicologico e psicosociale, mirato al mantenimento/modellamento delle modalità interattive con i pari e, alla costruzione e riconfigurazione delle possibilità/ capacità relazionali. In questo senso lo strumento del gruppo omogeneo ${ }^{15}$ sosterrebbe detta riconfigurazione, contenendo e permettendo la successiva elaborazione, in un "luogo sicuro" e in una prima fase post terapia, di quanto vissuto durante le varie fasi della cura.

Il Convegno ha avuto il valore aggiunto di essere una reale occasione di incontro dialogico operatori-pazienti, e di divenire rappresentativo del fatto che uno spazio protetto e dedicato all'ascolto interessato costituisca il primo passo per una buona e costruttiva comunicazione.

L'indagine in plenaria, pur non avendo la caratteristica della riservatezza, ha permesso (per l'alto grado di interesse, attenzione e partecipazione attiva degli intervistati) un clima di condivisione che ha favorito in molti ragazzi (quasi il 90\% dei presenti) il desiderio e l'occasione di esprimersi dinanzi a tutti gli attori citati nelle dinamiche di comunicazione sulla malattia: medici, infermieri, psicologi, compagni di percorso, volontari e familiari. Ha restituito ciò che di solito viene riferito in modo più esclusivo al singolo professionista. 


\section{LIMITI DELLO STUDIO E PROSPETTIVE}

Data la libertà di partecipazione, il campione potrebbe non essere stato rappresentativo dell'intero universo dei vissuti esperienziali oggetto di interesse ${ }^{7}$. Il raggiungimento della sede congressuale (Roma), lo stato clinico della contingenza temporale specifica e la disposizione caratteriale dei singoli a parlare in pubblico, potrebbero aver favorito la presenza e l'eloquio di alcuni rispetto ad altri e offerto una sintesi dalle sfumature non sempre statisticamente condivisibili. Inoltre, la motivazione alla presenza e alla partecipazione possono aver involontariamente selezionato gli intervenuti come gruppo di pazienti che, rispetto al tema, aveva particolare volontà di esprimere e/o raccontare la propria esperienza e i propri punti di vista.

Per quanto riguarda la mancanza di riservatezza propria di un ambito congressuale, possiamo avanzare l'ipotesi che il ritrovarsi riuniti in un numero considerevole, in uno spazio definito rispetto al resto della sala, e sottoposti a un compito comune, abbia condizionato il compito e allo stesso tempo permesso l'attivazione di una serie di dinamiche gruppali ${ }^{15}$, rintracciabili nella definizione generica di gruppo in "assunto di base"16. In questo senso, il gruppo ha probabilmente rappresentato un luogo sicuro di condivisione e di rispecchiamento primario tra pari che ha permesso ai singoli il recupero su base gruppale di parte del proprio narcisismo adolescenziale ferito a causa della diagnosi e delle modificazioni fisiche conseguenza della malattia o delle terapie. Infine, la vivace spinta, a tratti "ironica", alla partecipazione di gruppo e la voglia di condivisione della maggior parte dei ragazzi del gruppo allargato romano, spinge a continuare la ricerca nel campo dell'applicazione dello strumento gruppale in ambiti alternativi a quelli clinici classici (ospedalieri o da studio). Tali ambiti, nonostante i difetti rilevati, permettono, per numero e dinamiche di gruppo allargato, la partecipazione e lo studio di movimenti gruppali molto interessanti non altrimenti ripetibili.

\section{Messaggi chiave}

- Gli adolescenti con tumore rappresentano un gruppo particolare di pazienti non riconducibile all'Oncologia pediatrica o dell'adulto, anche per il significato attribuito alle relazioni tra pari.

- Vi è la necessità, per questo gruppo di pazienti, di attuare progetti dedicati in termini di spazi e di personale specificatamente formato, tale da attendere alla vita relazionale dei pazienti come a una tra le priorità.

- La qualità di vita del giovane paziente non può eludere le dinamiche amicali, per le quali è richiesto un supporto per il rimodellamento delle possibilità/capacità relazionali.

\section{*Gruppo di Lavoro Adolescenti AIEOP Associazione Italiana Emato-Oncologia Pediatrica}

Maurizio Mascarin ${ }^{1}$, Francesca Bomben ${ }^{1}$, Marina Bertolotti ${ }^{2}$, Teresa Perillo $^{3}$, Marta Pierobon $^{4}$, Paola Quarello $^{2}$, Domitilla Elena Secco ${ }^{5}$, Marco Spinelli ${ }^{6}$, Assunta Tornesello ${ }^{7}$, Paolo Colavero, Federico Mercolini, Antonella Guido ${ }^{9}$, Silvia Pomi ${ }^{10}$, Laura Guidotti ${ }^{10}$, Daniele Zama ${ }^{11}$, Maria Concetta Galati ${ }^{12}$, Luca Coccoli ${ }^{13}$, Celeste Ricciardi ${ }^{14}$, Giuseppe Robustelli ${ }^{15}$, Maria Speranza Massei ${ }^{16}$, Rosanna Martin ${ }^{17}$, Massimo Conte ${ }^{18}$, Marco Zecca ${ }^{19}$, Carlo Alfredo Clerici ${ }^{20}$, Andrea Ferrari ${ }^{21}$, Giuseppe Maria Milano ${ }^{5}$

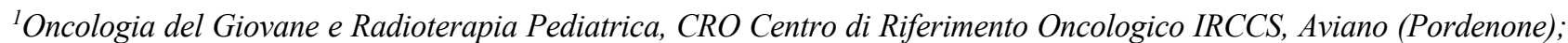
${ }^{2}$ Emato-Oncologia Pediatrica, AOU Città della Salute e della Scienza, Ospedale Regina Margherita, Torino; ${ }^{3}$ EmatoOncologia Pediatrica, Dipartimento di Pediatria, Università di Bari; ${ }^{4}$ Emato-Oncologia Pediatrica, Fondazione Città della Speranza, Università di Padova; ${ }^{5}$ Emato-Oncologia Pediatrica, Ospedale Pediatrico Bambino Gesù IRCCS, Roma; ${ }^{6}$ Emato-Oncologia Pediatrica e Centro Ricerche "Tettamanti", Università Milano-Bicocca, Fondazione MBBM, Ospedale San Gerardo, Monza; ${ }^{7}$ Oncologia Pediatrica, Ospedale Vito Fazzi, Lecce; ${ }^{8}$ Pediatria, Ospedale Centrale di Bolzano; ${ }^{9}$ Oncologia Pediatrica, Fondazione Policlinico Universitario A. Gemelli IRCCS, Roma; ${ }^{10}$ Onco-Ematologia Pediatrica. Ospedale dei Bambini di Parma; ${ }^{11}$ Oncologia, Ematologia e Trapianto di CSE - Pediatria - Policlinico di S. Orsola, Bologna; ${ }^{12}$ Emato-Oncologia Pediatrica A.O. Pugliese-Ciaccio Catanzaro; ${ }^{13}$ Onco-Ematologia Pediatrica, Ospedale S. Chiara, Pisa; ${ }^{14}$ Onco-Ematologia Pediatrica, Fondazione Casa Sollievo della Sofferenza IRCCS, San Giovanni Rotondo (Foggia) ${ }^{15}$ Onco-Ematologia Pediatrica, Dipartimento della Donna e del bambino, ASST Sette Laghi, Varese; ${ }^{16}$ Onco-Ematologia Pediatrica, Ospedale Santa Maria della Misericordia, Perugia; ${ }^{17}$ Psicologia Ospedaliera Pediatrica, Ospedale Meyer, Firenze; ${ }^{18}$ Oncologia, Istituto Giannina Gaslini, Genova; ${ }^{19}$ Emato-Oncologia Pediatrica, Fondazione IRCCS Policlinico San Matteo, Pavia; ${ }^{20}$ Scienze biomolecolari e biotecnologia, Sezione di Psicologia, Facoltà di Medicina, Università di Milano; ${ }^{21}$ Oncologia Pediatrica, Fondazione IRCCS Istituto Nazionale dei Tumori, Milano 


\section{BIBLIOGRAFIA}

[1] AIRTUM Working Group. I tumori infantili in Italia. Epidemiol Prev 2008;32:1-112.

[2] Ferrari A, Thomas D, Franklin AR, et al. Starting an adolescent and young adult program: some success stories and some obstacles to overcome. J Clin Oncol 2010;28(32):4850-7. DOI: 10.1200/JCO.2009.23.

[3] Mascarin M, Truccolo I, Byther E, Capone D, Elia C. Cancer, adolescence, and their peers: “They'll give you a story". J Cancer Educ 2014;29:434-40. DOI: $10.1007 / \mathrm{s} 13187-014-0635-9$.

[4] Ferrari A, Clerici CA, Casanova M, et al. The Youth Project at the Istituto Nazionale Tumori in Milan. Tumori 2012;98(4):399-407. DOI: 10.1700/ 1146. 12631.

[5] Ferrari A, Rondelli R, Pession A, et al. Adolescents with cancer in Italy: improving access to National Cooperative Pediatric Oncology Group (AIEOP) Centers. Pediatr Blood Cancer 2016;63(6):1116-9. DOI: 10.1002/pbc.25954.

[6] Institute of Medicine. Identifying and addressing the needs of adolescents and young adults with cancer: workshop summary. Washington, DC: The National Academies Press, 2013. DOI: 10.17226/ 18547.

[7] Bertolotti M. Aspetti psicologici: non per tutti lo stesso percorso. I tumori in Italia. Rapporto AIRTUM 2012, Tumori Infantili. Epidemiol Prev 2013;37(1) suppl 1:1-296.

[8] Proserpio T, Veneroni L, Silva M, et al. Spiritual support for adolescent cancer patients: a survey of pediatric oncology centers in Italy and Spain. Tumori 2016;102(4):376-80. DOI: 10.5301/tj. 5000494.
[9] Schultz KA, Ness KK, Whitton J, et al. Behavioral and social outcomes in adolescent survivors of childhood cancer: a report from the childhood cancer survivor study. J Clin Oncol 2007;25(24): 3649-56. DOI: 10.1200/JCO.2006.09.2486.

[10] Bolasco S. Analisi multidimensionale dei dati. Metodi, strategie e criteri d'interpretazione. Roma: Carocci, 1999.

[11] Samuel CA, Mbah O, Schaal J, et al. The role of patient-physician relationship on health-related quality of life and pain in cancer patients. Support Care Cancer 2020;28(6):2615-26. DOI: $10.1007 /$ s00520-019-05070-y.

[12] Magnezi R, Bergman LC, Urowitz S. Would your patient prefer to be considered your friend? Patient preferences in physician relationships. Health Educ Behav 2015;42(2):210-9. DOI: 10.1177/ 1090198114547814.

[13] Siembida EJ, Bellizzi KM. The doctor-patient relationship in the adolescent cCancer setting: a developmentally focused literature review. J Adolesc Young Adult Oncol 2015;4(3):108-17. DOI: 10. 1089/jayao.2015.0011.

[14] Rugarli C. Medici a metà. Quel che manca alla relazione di cura. Milano: Raffaello Cortina Editore, 2017.

[15] Marinelli S. Funzioni dell'omogeneità nel gruppo. In: Corbella S, R. Girelli R, Marinelli S (a cura di). Gruppi omogenei. Teoria e tecnica del campo mentale omogeneo. Roma: Borla, 2004, pagg. 4162.

[16] Bion WR. Esperienze nei gruppi. Roma: Armando, 1971. 